Research Article

\title{
The Scale Effect of Coarse-Grained Materials by Triaxial Test Simulation
}

\author{
Xiaotao Ai $\mathbb{D},{ }^{1}$ Guangjin Wang $\mathbb{D}^{1,2,3,4}$ Xiangyun Kong $\mathbb{D}^{1,2,4}$ Bo Cui ${ }^{1}{ }^{1}{ }^{1}$ Bin $\mathrm{Hu}(\mathbb{D})^{5}$ \\ and Hongling $\mathrm{Ma}^{3}{ }^{3}$ \\ ${ }^{1}$ Faculty of Land Resources Engineering, Kunming University of Science and Technology, Kunming 650093, China \\ ${ }^{2}$ National and Local Joint Engineering Research Center for Green Comprehensive Utilization of Metal Ore Tailings Resources, \\ Kunming 650093, China \\ ${ }^{3}$ State Key Laboratory of Geomechanics and Geotechnical Engineering, Institute of Rock and Soil Mechanics, \\ Chinese Academy of Sciences, Wuhan 430071, China \\ ${ }^{4}$ Yunnan Key Laboratory of Sino-German Blue Mining and Utilization of Special Underground Space, Kunming 650093, China \\ ${ }^{5}$ School of Resources and Environmental Engineering, Wuhan University of Science and Technology, Wuhan 430081, China
}

Correspondence should be addressed to Guangjin Wang; wangguangjin2005@163.com and Xiangyun Kong; 12643015@qq.com

Received 16 November 2020; Revised 21 December 2020; Accepted 23 December 2020; Published 18 January 2021

Academic Editor: Chong Xu

Copyright (c) 2021 Xiaotao Ai et al. This is an open access article distributed under the Creative Commons Attribution License, which permits unrestricted use, distribution, and reproduction in any medium, provided the original work is properly cited.

The scale effect is an unavoidable problem in the laboratory test of coarse-grained materials. By combining the self-developed cellular automaton program with laboratory experiments, a method of simulating the triaxial test of coarse-grained materials was proposed in this paper, and a triaxial test numerical specimen that can characterize the discontinuous, nonuniform, and heterogeneous characteristics of bulk geotechnical materials was established. The parallel grading method was adopted to create six grading curves for numerical simulation based on one in situ grading curve. The failure process and the scale effect on the strength and deformation of coarse-grained materials were analyzed and discussed. The results showed that under the same confining pressure, the peak stress and initial deformation modulus $E_{i}$ increased with the increase of the maximum particle size $d_{\max }$, while the degree of shear shrinkage and Poisson's ration $v$ decreased. As the confining pressure increased, the scale effect of coarse-grained materials would be magnified. If particle breakage and migration were assumed to be neglected, the internal friction angle $\varphi$ and $d_{\max }$ would be roughly proportional, the cohesive force $c$ fluctuated with the increase of $d_{\max }$, and the empirical relations between $d_{\max }$ and $c$ and $\varphi$ were established, respectively, which provides a reference for estimating the actual shear strength parameters of coarse-grained materials on-site. The research results can provide a way of thinking for the study of the scale effect of coarse-grained materials and also have certain reference significance for inferring the strength parameters of the original-graded coarse-grained materials.

\section{Introduction}

Because of good engineering properties, coarse-grained materials are widely used as the primary filling materials for railway ballasts, reservoirs, and dams and are also the main bulk geotechnical material piled up in the dumps of open-pit mines. Obtaining reasonable strength parameters of geomaterials is the primary task of studying the stability of such geotechnical engineering [1-8]. However, in practical engineering, the reasonable strength parameters of coarsegrained materials have always been a problem. Generally, the shear strength parameters of coarse-grained materials can be obtained through large-scale direct shear tests or large-scale triaxial tests. However, as a granular loose geotechnical material, the strength of coarse-grained materials is affected by gradation composition, particle shape, and intergranular force, mineral composition of rock, and other factors [9], so even coarse-grained material subjected to multiple same laboratory tests has the same gradation composition, and the results may not be consistent. Besides, the particle size of coarse-grained materials is very dispersed, and the size of large particles even exceeds $1 \mathrm{~m}$. To determine the shear 
strength parameters of such coarse-grained materials, a giant instrument with a diameter of more than 5 meters and a height of more than 10 meters needed to be established, and its size is far beyond the size of existing instruments [10]. The current method of studying this type of coarse-grained material is to reduce the gradation of the natural materials and try to estimate the actual strength parameters by measuring the strength parameters of the reduced coarsegrained materials. Because the scale reduction changes the gradation of the original coarse-grained materials, the physical and mechanical properties of the coarse-grained materials are also significantly changed, which is the scale effect.

The scale effect is mainly due to the fact that the scale changes the gradation composition of the coarse-grained materials, and the gradation is an essential factor affecting the physical and mechanical properties of the coarse-grained materials. Many scholars have studied the relationship between the gradation and the physical and mechanical properties of coarse-grained materials. Wei et al. [11] found that the increase of the coarse coarse-grained content will increase the strength of the coarse-grained material, and the degree of particle breakage will increase accordingly. Zhang et al. [12] believed that with the increase of the coarsegrained content, the permeability coefficient, and internal friction angle of the coarse-grained material tends to increase. Huang et al. [13] found that the effect of gradation on the stress-strain curve and volume strain-axial strain curve of coarse-grained materials is more significant under the condition of low confining pressure. Through the thermal needle test, Xiao et al. [14] found that the thermal conductivity of sand increased with the increase of the variable coefficient of the gradation curve. Besides, some scholars have conducted research on the factors influencing the scaling effect of coarse-grained materials. Yang et al. [15] studied the influence of particle gradation on the critical state of rockfill materials and found that when the combination method was used to scale coarse-grained materials, the critical state stress ratio increased with the increase of the maximum particle size. Wei et al. [16] found that when the same scaling method is used, the compressive modulus will increase with the increase of the maximum particle size. Hamidi et al. [17] studied the shear strength and dilatancy of coarse-grained materials based on 27 large-scale direct shear tests and established the empirical formula of estimating the shear strength parameters of the parallel grading method.

At present, most of the research methods for coarsegrained materials are based on laboratory tests. Numerical simulation can make up for the lack of workforce, material resources, and energy in the laboratory test and is an effective means to supplement the laboratory test of coarse-grained materials. However, most of the numerical simulation of coarse-grained materials study the effect of gradation on the physical and mechanical properties based on the particle flow code software PFC $[18,19]$, but few studies on the scaling effect. The scale effect is an unavoidable phenomenon in the current study of coarsegrained materials. In order to better understand the physical and mechanical properties of the original-graded coarse-grained materials, it is necessary to strengthen the research on the scale effect.

Compared with the PFC in which microscopic parameters needs to be determined by adjusting the parameters, the macromechanical parameters in the fast Lagrangian analysis of continua software FLAC can be obtained relatively reliably according to the laboratory test. If new analysis methods are introduced to make the numerical simulation calculation of coarse-grained materials possible through FLAC, it can also provide an idea for the numerical simulation of coarse-grained materials. Cellular automata (CA) are a grid dynamic model with discrete and interacting states and time and space. It has stronger computing power than general mathematical models and a high degree of dynamic and spatial concepts. After CA was proposed, researchers have been trying to apply it to their research and achieved many reliable results. However, most of them focused on rock bursts and rock fractures [20], soil particle size distribution [21], element migration in the soil [22], and other aspects, and only a few scholars [23-25] applied CA into the numerical simulation research of coarse-grained materials.

In this paper, based on laboratory experiments, by combining the self-compiled cellular automata program SEPOHM with numerical calculation software FLAC ${ }^{3 \mathrm{D}}$, a three-axis numerical simulation method for coarse-grained materials was proposed. This method can effectively characterize the random and uneven distribution of particles in each group of coarse-grained materials and efficiently complete the simulation of the triaxial test of coarse-grained materials. It can also eliminate the influence of the human factor on the test results during sample preparation. Aiming at the coarse-grained materials accumulated in a dumping site of a copper mine in Jiangxi, China, six numerical samples of coarse-grained materials with different reducedscale ratios were prepared using the parallel grading method, and the scale effect of the coarse-grained materials was studied. By analyzing the stress-strain curve of the tested coarse-grained material, some common understandings have been obtained, which make us better understand the mechanical behavior of the coarse-grained material during the triaxial test and can also provide a certain reference for actual engineering.

\section{Simulation of the Triaxial Test and Its Validity Verification}

Coarse-grained materials have the characteristics of discontinuity, nonuniformity, and heterogeneity in the microscopic view. The connection state between the granular particles with different sizes causes the discontinuity of the coarse-grained material. Regardless of the connection state between the particles, the deformation will be preferentially generated at the particle contact surface under the action of external force and cause damage such as tensile cracking, slippage, and embedding, which leads to the discontinuously distributed stress field and displacement field. Coarsegrained materials are composed of granular particles with different sizes and strengths, which make the coarse-grained materials have nonuniform characteristics. The random 
distribution of loose particles and the difference in gradation and particle geometry make the coarse-grained materials show heterogeneity.

This paper used the software SEPOHM and FLAC ${ }^{3 \mathrm{D}}$ to discretize the calculation model of the triaxial specimen according to the particle size distribution of the coarsegrained material. The difference in mechanical properties of different granular groups reflects the discontinuous, inhomogeneous, and heterogeneous characteristics of the coarse granular material from the mechanical point of view. The initial structure of the coarse-grained materials was qualitatively simulated to realize the anisotropy of the strength of the coarse-grained numerical specimens.

2.1. Cellular Automata SEPOHM. As for cellular automata (CA), complex mathematical rules can be avoided, and the entire system can evolve only by partial and straightforward rules. In order to simulate the random and inhomogeneous spatial characteristics of each granule group, a cellular automata program was developed and named as the stochastic evolution program of heterogeneous materials (SEPOHMs). This program can evolve the structure of the coarse-grained sample with the specified gradation by defining the content of the coarse-grained material in different particle groups. Because of the temporal and spatial discrete characteristics of CA, the program SEPOHM can randomly generate samples of the same gradation coarse-grained material with the different distribution of each particle group to consider the structural characteristics of the coarsegrained material. At the same time, the program also has the advantages of simple operation and fast calculation speed and realizes the visualization of the distribution of each granule group.

Cellular automata are composed of four systems: cell, neighbour cell, cellular space, and evolution rule. A cellular automaton is a program that evolves cells in different states according to specific rules in the cellular space. The cell is the most basic unit that composes cellular automata, which are distributed in discrete cellular space. The cellular space is the space in which the cell evolves. It can be one-dimensional, two-dimensional, or multidimensional Euclidean space. There are a certain number of discrete lattice points distributed in this space, and each lattice point can accommodate a cell. The basic structure of the software SEPOHM is shown in Figure 1.

It can be seen from Figure 1 that SEPOHM uses Mooretype neighbours including the top, bottom, left, right, top right, bottom right, top left, and bottom left, in which bottom left of the central cell is the neighbour cells. Besides, there are five kinds of cell states in SEPOHM, which are represented by the numbers $0,1,2,3$, and 4 . At the same time, cells of different states are given different colours. In theory, the cell space is infinitely stretched, but this ideal state cannot be realized under the current calculation conditions. Therefore, in the actual operation process, the cellular automaton has boundaries. SEPOHM adopts periodic boundary conditions, as shown in Figure 2, which regards the relative boundaries of the cell space as connected.

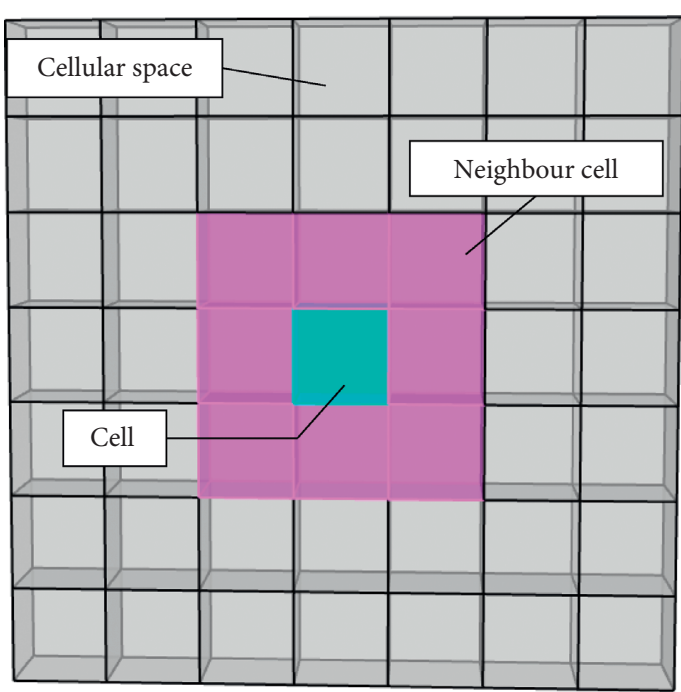

FIGURE 1: Schematic diagram of the basic structure of SEPOHM.

In the two-dimensional space, cellular space appears as a topological ring connected head to tail, which is the closest to infinite space. Therefore, periodic boundary conditions are usually used in the development of cellular automata.

The software SEPOHM uses the eight-neighbour model and periodic boundary conditions. There are five states of cells representing the five granular groups of coarse-grained materials, and different colours were used to distinguish cells at different states. The rules of the software are as follows: after setting the percentage of the five materials, the cells in the four states are first randomly generated in the evolution space according to the time-related function, and after reaching the respective content requirements set by the system, it stops evolving. At this time, the fifth cell is generated in the space of the ungenerated cells. Figure 3 shows a schematic diagram of the software SEPOHM that completes the evolution after entering the specified content of each granule group. Taking the grid of the FLAC ${ }^{3 \mathrm{D}}$ calculation model of the test piece as the evolution space of the software SEPOHM, a grid in FLAC ${ }^{3 \mathrm{D}}$ is a cellular space in SEPOHM, and the distribution of each particle group evolved by the software can be imported into the established FLAC $^{3 \mathrm{D}}$ model of the sample, and five numerical sample models for the triaxial compression test of coarse-grained materials with random and uneven distribution of particle groups are generated.

2.2. Large-Scale Triaxial Compression Test. In order to guide the numerical simulation of coarse-grained materials, a largescale triaxial test was carried out (Figure 4) on a $1500 \mathrm{KN}$ electrohydraulic servo dynamic and static triaxial test machine in the Wuhan Institute of Geotechnical Engineering, Chinese Academy of Sciences. The sample for this test is a soil-rock mixed coarse-grained material accumulated in a dumping site of a copper mine in Jiangxi, China (Figure 5). A sampling at the middle of the side slope of the dump, the maximum particle size of the soil sample is $300 \mathrm{~mm}$. The gradation can be seen in Figure 6 as "natural gradation." The 


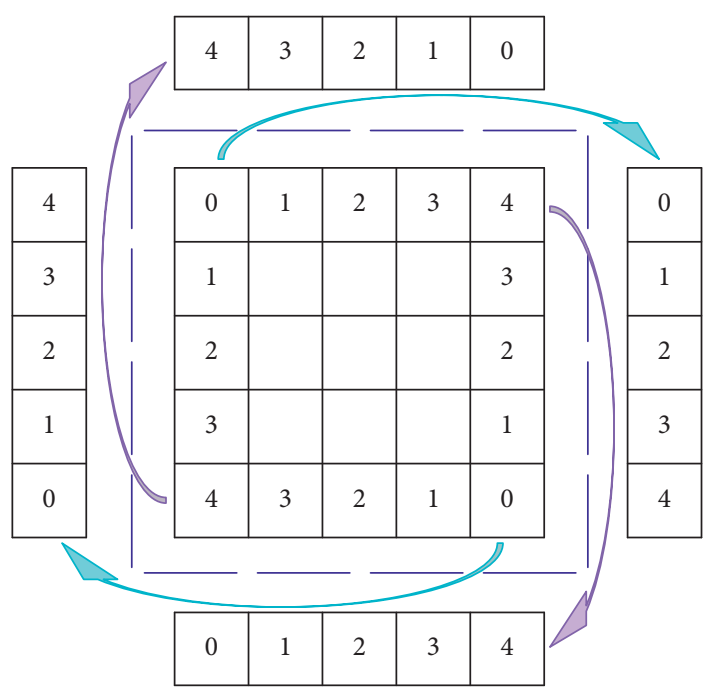

FIgURE 2: Schematic diagram of periodic boundary conditions.



FIGURE 3: Schematic diagram of software SEPOHM.

size of the test piece is $0.3 \mathrm{~m}$ (diameter) $\times 0.6 \mathrm{~m}$ (height). Four confining pressures of $400 \mathrm{kPa}, 800 \mathrm{kPa}, 1200 \mathrm{kPa}$, and $1600 \mathrm{kPa}$ are designed, respectively, and the shear rate is $5 \times 10^{-7} \mathrm{~m} / \mathrm{s}$. If the axial strain of the specimen exceeds $15 \%$, the specimen is thought to be damaged, and the test stopped.

The sample model of the triaxial simulation test is shown in Figure 7. The model size was $3 \mathrm{~m}$ (diameter) $\times 6 \mathrm{~m}$ (height) The boundary conditions were fixed bottom surface $(z=0 \mathrm{~m})$, the constant velocity $v$ of the top surface $(z=6 \mathrm{~m})$, and confining pressure $\sigma$ on the side of the cylinder. To ensure the simulated large-scale triaxial test is consistent with the actual situation, the simulated axial strain rate $v$ was equal to $5 \times 10^{-6} \mathrm{~m} / \mathrm{s}$, and the confining pressure $\sigma$ of each group was $400 \mathrm{kPa}, 800 \mathrm{kPa}, 1200 \mathrm{kPa}$, and $1600 \mathrm{kPa}$, respectively.

According to Q. G. Guo's research on coarse-grained materials [26] and the geotechnical standard sieve diameter and considering the controlled particle size of the large triaxial instrument and the actual research needs in this paper, large-scale triaxial tests were carried out on the coarse-grained soil samples with seven different grain groups $(d \leq 5 \mathrm{~mm}, 5 \mathrm{~mm}<d \leq 10 \mathrm{~mm}$, $10 \mathrm{~mm}<d \leq 20 \mathrm{~mm}, 20 \mathrm{~mm}<d \leq 40 \mathrm{~mm}, 40 \mathrm{~mm}<d \leq 60 \mathrm{~mm}$, $20 \mathrm{~mm}<d \leq 30 \mathrm{~mm}$, and $40 \mathrm{~mm}<d \leq 50 \mathrm{~mm}$ ) after screening the on-site coarse-grained material. The mechanical parameters of each granule group obtained are listed in Table 1 .

According to the grading composition of the sample, the contents of the five particle groups were set in the software SEPOHM, and then the software evolution results were introduced into the numerical model of the sample, as shown in Figure 8. It can be seen from Figure 8 that the numerical specimens established by software SEPOHM can well represent the random and discontinuous distribution state of each coarse particle group and the geometric shape of "cluster," indicating that it is feasible to simulate the distribution of coarse particle by software SEPOHM.

Based on the material parameters of each granular group in Table 1, the Mohr-Coulomb model was used to simulate the triaxial test of coarse granule material, and the simulated test results were compared with the actual laboratory test results. The stress-strain relationship is shown in Figure 9(a), and the relationship between volumetric strain $\varepsilon_{v}$ and axial strain is shown in Figure 9(b); $\varepsilon_{v}$ decreases to positive. It can be seen that the simulated triaxial test results are roughly consistent with the laboratory test results, indicating that the simulation method of the large-scale triaxial test adopted in this paper can basically reflect the macroscopic mechanical properties and deformation characteristics of coarse-grained materials.

\section{Introduction of the Test Scheme}

In this paper, the software SEPOHM can be used to prepare numerical samples with different soil particle structure to establish a virtual triaxial numerical simulation laboratory for coarse-grained material.

It is usually necessary to scale the original coarse granule material because the size of the large granule of the coarse granule material exceeds the maximum allowable size of existing instruments and equipment. At present, there are four commonly used scaling methods including knockout method, equal replacement method, parallel grading method, and combination method. Among them, the parallel grading method refers to the reduction of coarse particles according to the geometric similarity conditions, which can be expressed by formula (1). The grading curve using this method can keep the nonuniform coefficient $\mathrm{Cu}$ and curvature coefficient $C c$ of coarse granule materials unchanged and retain the characteristics of the original grading curve: 




(a)

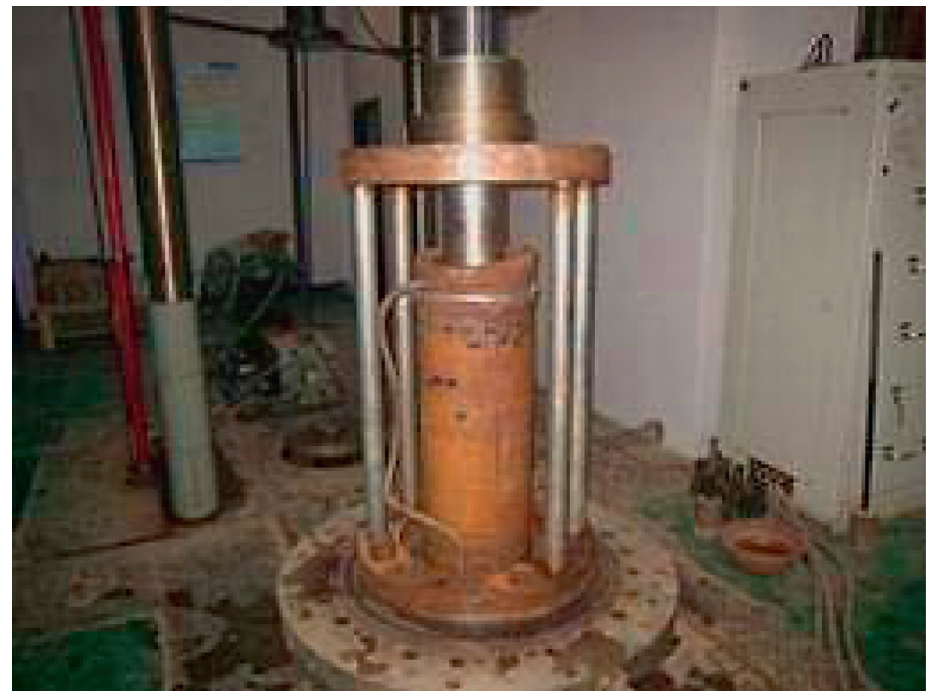

(c)



(b)



(d)

FIgURE 4: Large-scale triaxial test of coarse-grained materials: (a) laboratory apparatus; (b) installation sample; (c) before the test; (d) after the test.

$$
\begin{aligned}
d_{n i} & =\frac{d_{o i}}{n}, \\
P_{d n} & =\frac{P_{d o}}{n}, \\
n & =\frac{d_{o \max }}{d_{\max }},
\end{aligned}
$$

where $d_{n i}$ is one particle size after scaling, $\mathrm{mm} ; d_{o i}$ is one particle size of the original grading, $\mathrm{mm} ; d_{\text {omax }}$ is the maximum particle size of the initial grading, $\mathrm{mm} ; d_{\max }$ is the maximum particle size after scaling, $\mathrm{mm} ; P_{d n}$ is the content of particle less than certain size after $n$ times reduction, \%; and $P_{d o}$ is the content of the original grading less than certain particle size, $\%$.

In order to explore the influence of scale effect on the mechanical properties of coarse-grained materials, the 


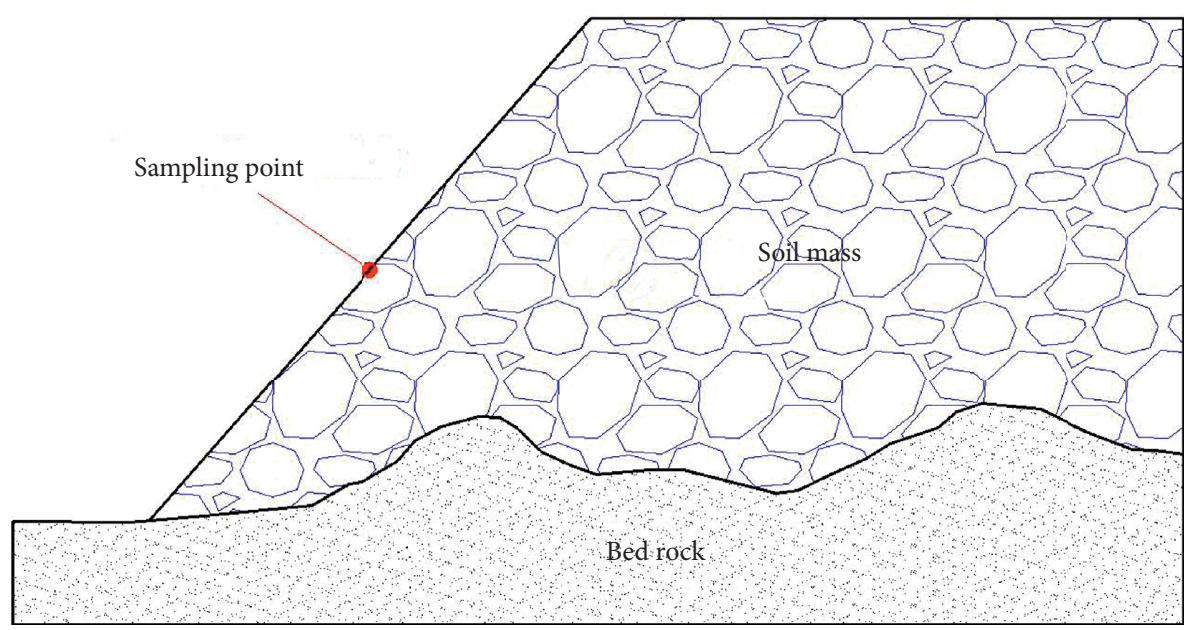

(a)

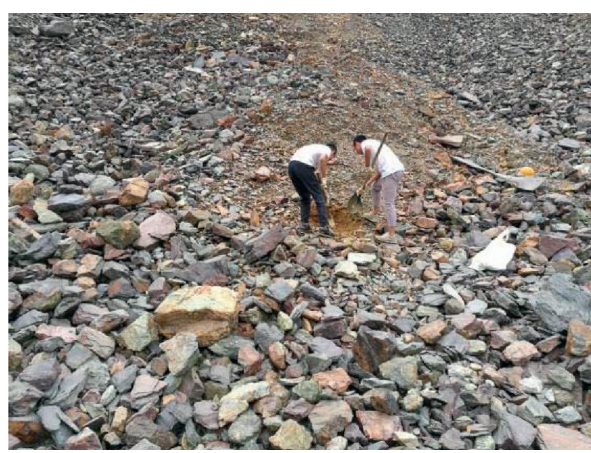

(b)

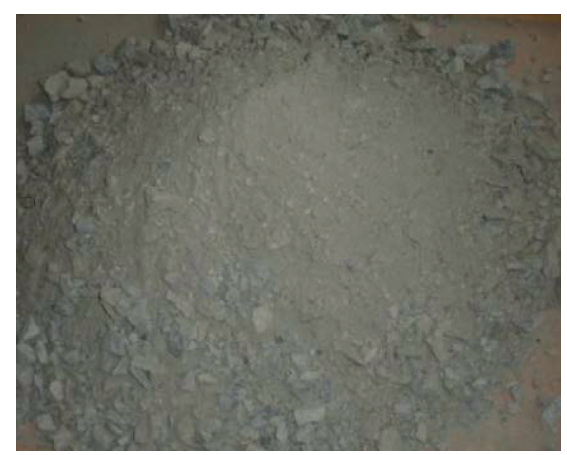

(c)

FIGURE 5: Coarse-grained materials accumulated in dump: (a) the location of sampling; (b) field sampling; (c) soil sample for test.

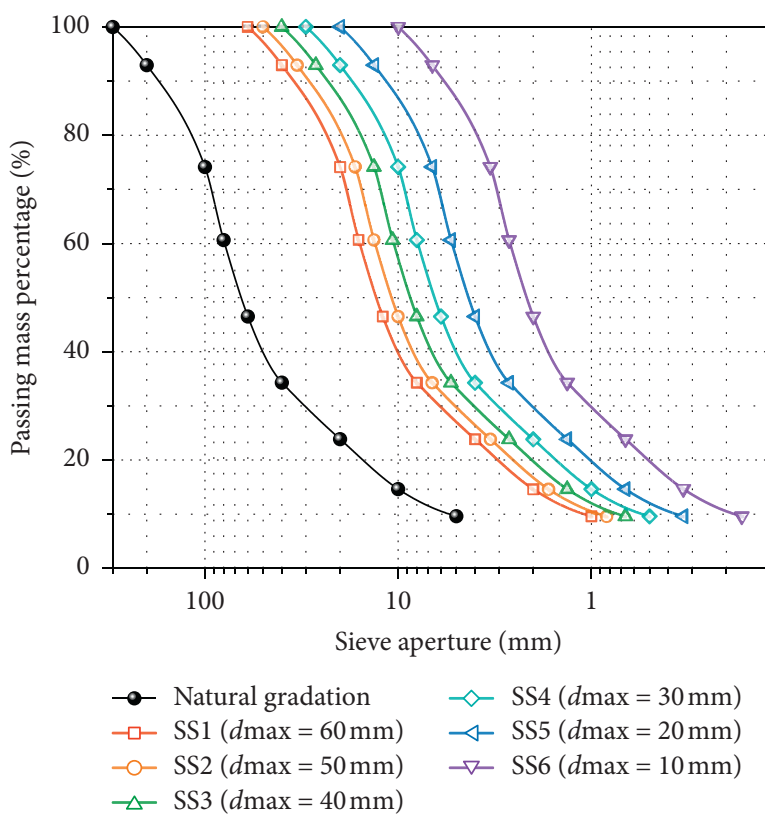

FIGURE 6: Grading curves of each scheme.

parallel grading method was adopted to reduce the bulk of the original-graded blasting materials into coarse-grained materials with maximum particle sizes $d_{\max }$ of $60 \mathrm{~mm}$,

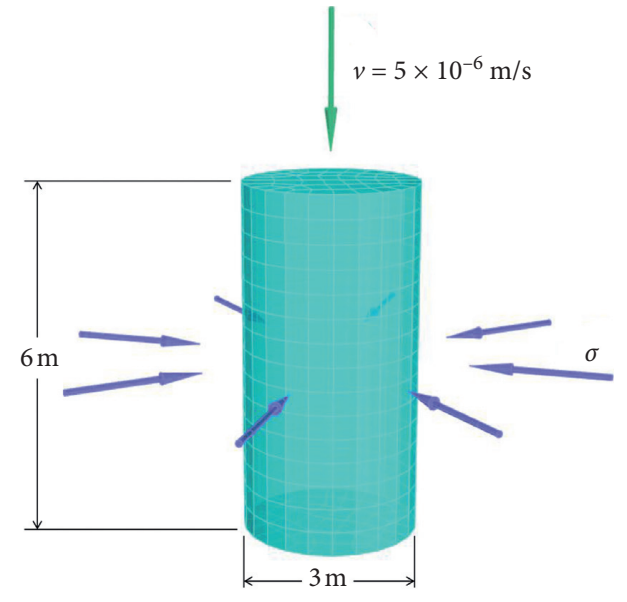

Figure 7: Schematic diagram of the numerical specimen model.

$50 \mathrm{~mm}, 40 \mathrm{~mm}, 30 \mathrm{~mm}, 20 \mathrm{~mm}$, and $10 \mathrm{~mm}$ in a copper mine dump in Jiangxi, China, namely, scheme SS1, scheme SS2, scheme SS3, scheme SS4, scheme SS5, and scheme SS6. The grading curve of each scheme is shown in Figure 8, and it can be seen that the grading curves of the original grading are smooth and concave with a gentle slope and continuous grading. At the same time, in order to consider the different situations of the coarse-grained material structure and make 
TABLE 1: The value of the mechanical parameters of each granule group.

\begin{tabular}{lcccccc}
\hline Granule group $(\mathrm{mm})$ & $\rho\left(\mathrm{g} \bullet \mathrm{cm}^{-3)}\right.$ & $K(\mathrm{Mpa})$ & $G(\mathrm{Mpa})$ & $\sigma_{t}(\mathrm{kPa})$ & $c(\mathrm{kPa})$ & $\varphi\left(^{\circ}\right)$ \\
\hline$d \leq 5$ & 1.85 & 35 & 10 & 0 & 39.1 & 21.7 \\
$5<d \leq 10$ & 1.91 & 70 & 30 & 0 & 26.2 & 22.6 \\
$10<d \leq 20$ & 2.10 & 120 & 75 & 0 & 34.7 & 24.2 \\
$20<d \leq 40$ & 2.21 & 260 & 105 & 0 & 52.3 & 29.4 \\
$40<d \leq 60$ & 2.28 & 700 & 310 & 0 & 86.0 & 32.5 \\
$20<d \leq 30$ & 2.16 & 175 & 95 & 0 & 44.1 & 27.6 \\
$40<d \leq 50$ & 2.25 & 540 & 275 & 0 & 67.3 & 6.0 \\
\hline
\end{tabular}

Note. $\rho$, density; $K$, bulk modulus; $G$, shear modulus; $\sigma_{t}$, tensile strength; $c$, cohesion; $\varphi$, internal friction angle; $\psi$, dilatancy angle.

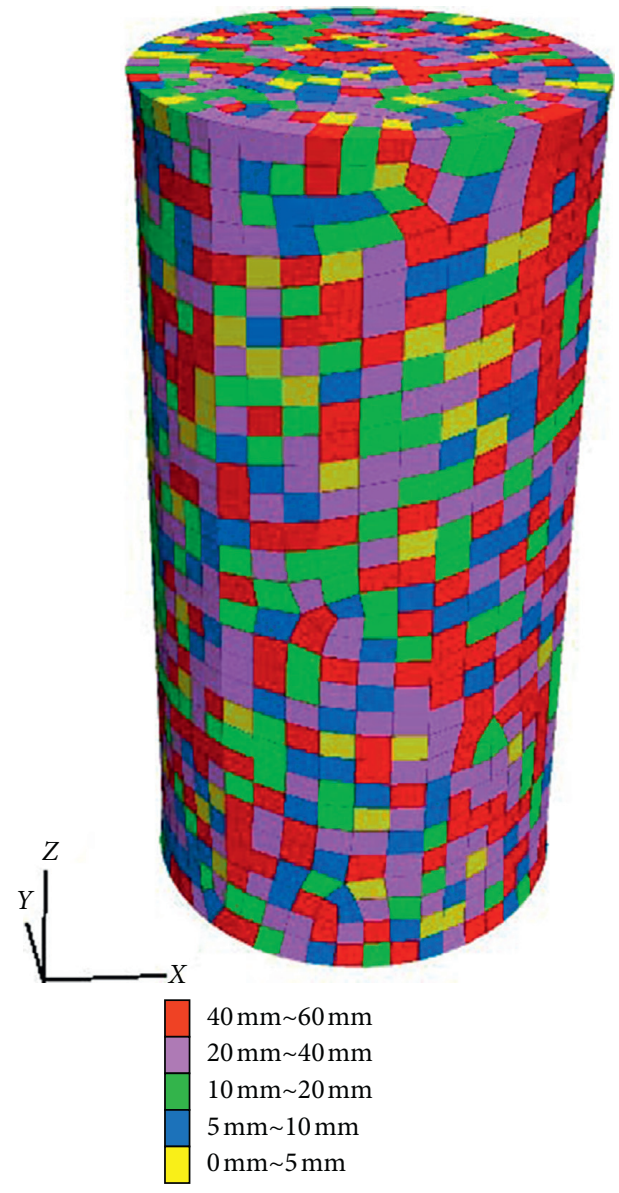

Figure 8: A schematic diagram of a triaxial numerical specimen.

the calculation results of each scheme more representative, twenty groups of numerical specimens were prepared for the coarse-grained material of each grading scheme. The contents of each group are the same, but the distribution is different. The triaxial compression simulation tests were carried out for each group under four different confining pressure. Therefore, 120 groups of numerical specimens and 480 triaxial compression simulation tests need to be prepared for the six schemes in this paper.

Since SEPOHM can specify up to five kinds of particle group contents with different particle size ranges, the corresponding relationships can be seen in Figure 10. The parameter values of each grain group during simulation can be seen in Table 1.

\section{Results and Discussion}

4.1. Stress-Strain Relationship under Different Confining Pressure Conditions. Figure 11 shows the stress-strain curves of each scheme obtained from a simulation test when $\sigma$ is $1600 \mathrm{kPa}$. It can be seen that the stress-strain curve is generally hardening, so the stress when the strain is $15 \%$ is taken as the peak stress. Under the same confining pressure, the peak stress decreased with the reduction of $d_{\max }$, indicating that with the increase of scale reduction degree, the strength of coarse-grained material decreased and the scale effect became more apparent. At the same time, the slope of the stress-strain curve of schemes SS1 and SS6 decreased successively, indicating that the initial deformation modulus $E i$ of coarse-grained material decreases with the increase of the scale ratio. This may be one of the reasons why the displacement calculated by the numerical simulation in the current research is usually smaller than the actual displacement. In actual engineering, the method of reducing the scale will not make the strength of the scaled material higher than that of the original grading coarse-grained material. This is because the scale discards the supersized particles, and the strength of the supersized particles is often greater than that of the fine particles. Although various scaling methods have adopted certain measures to compensate for the strength loss caused by the scale, most of them are difficult to reflect the strength of the originalgraded coarse-grained material fully.

4.2. Deformation Feature Analysis. According to Figure 9(b), it can be seen that the degree of shear shrinkage increases with the increase of the confining pressure. Figure 12 shows the volumetric strain-axial strain curve of the numerical samples of each scheme under the confining pressure of $1600 \mathrm{kPa}$, and it can be seen the volumetric strain decreases to positive, the coarse-grained materials of each scheme all undergo shear shrinkage under high-stress condition, and the shear shrinkage degree increases with the decrease of the maximum particle size $d_{\max }$. This may be due to the fact that the coarse-grained materials reduced by the parallel grading method increase with the decrease of $d_{\max }$, and the fine particle content increases. The fine particles can be better filled in the pores between the framework particles, and the strength of the fine particles is lower, which makes the shear shrinkage degree gradually increased.

In addition, the deformation characteristics of the triaxial specimen can also be reflected by the deformation 




(a)



(b)

Figure 9: Comparison of laboratory test results and simulation results: (a) stress-strain curve; (b) volumetric strain curve.

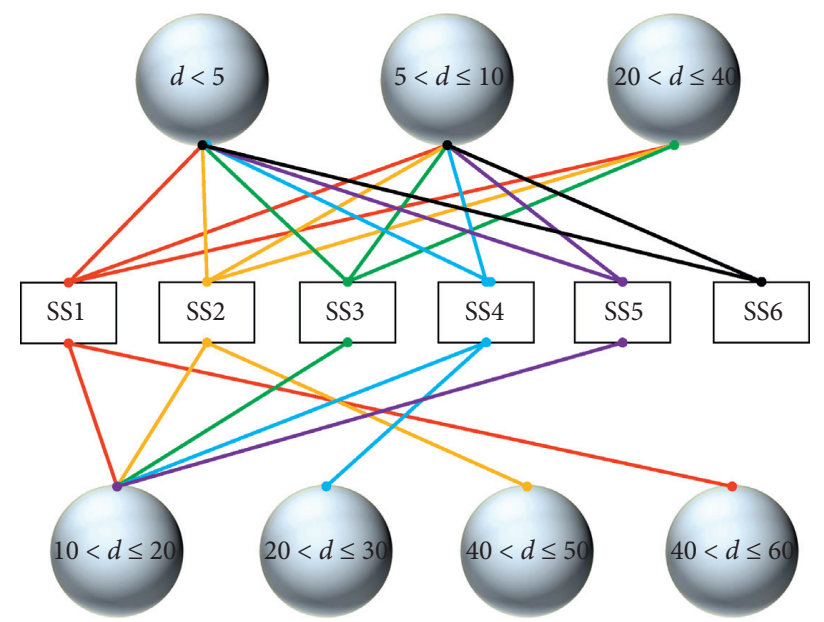

FIgURE 10: The particle size range of the granule group corresponding to each scheme (unit: $\mathrm{mm}$ ).

modulus $E$ and Poisson's ratio $v$. $E$ can be obtained from the deviator stress-axial strain curve, and its value is the secant modulus of the curve when it reaches half of the peak strength; the $v$ of the triaxial sample can be calculated from formula (2) according to the volume strain during the test:

$$
\begin{gathered}
\varepsilon_{r}=\frac{\varepsilon_{v}-\varepsilon_{a}}{2}, \\
\nu=-\frac{\varepsilon_{r}}{\varepsilon_{a}},
\end{gathered}
$$

where $\varepsilon_{r}$ is the radial strain, $\%$; $\varepsilon_{v}$ is the volume strain, \%; and $\varepsilon_{a}$ is the axial strain, \%.

When the confining pressure is $1600 \mathrm{kPa}$, the calculation results of $E$ and $v$ of the numerical samples of each scheme can be seen in Figures 13 and 14, respectively. It can be seen that $E$ gradually decreases with the decrease of the maximum particle size $d_{\max }$, and the relationship can be fitted with a linear relationship in Figure 13. As $d_{\max }$ decreases, the content of fine particles gradually increases, so that the filling relationship between coarse and fine particles gradually was improved to form a more stable and dense structure, so the axial deformation caused by the initial shear is gradually reduced. It can be seen from Figure 14 that the tangent Poisson's ratio of the numerical samples of each scheme under high-stress conditions has the same change, which increases with the increase of radial strain. The tangent Poisson's ratio has little difference in the initial stage. With the continuous increase of the axial strain, the difference of the tangent Poisson's ratio gradually increases, and the tangent Poisson's ratio gradually decreases with the increase of $d_{\max }$. The reason may be that the migration and fragmentation of particles were not considered during the simulation. Therefore, the numerical sample occurred elastic deformation at the beginning of the test, causing Poisson's ratio to be roughly the same in the initial stage. As the test 




FIgURE 11: Stress-strain curves of each scheme at $1600 \mathrm{kPa}$ confining pressure.

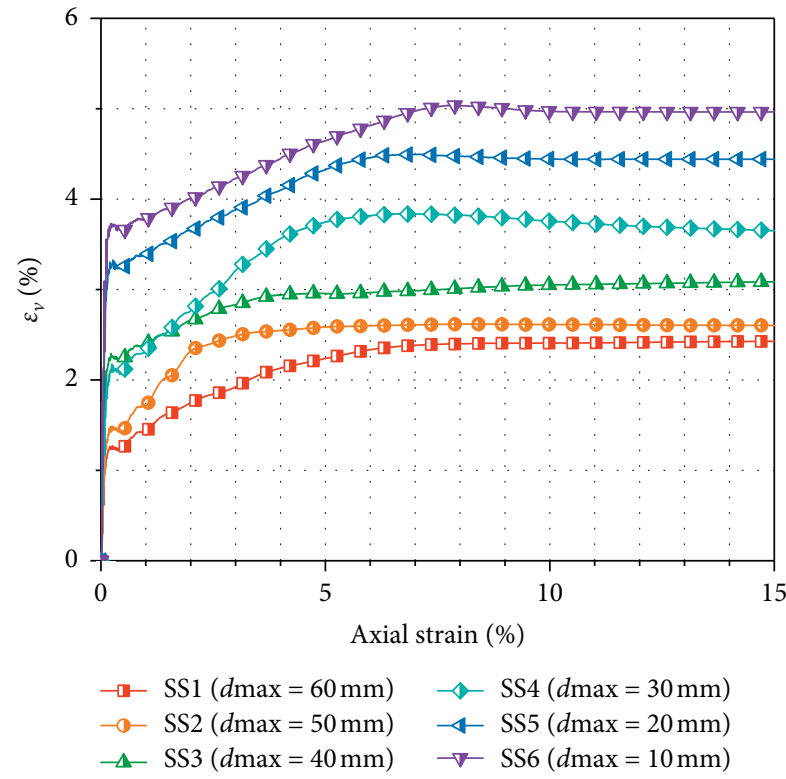

FIGURE 12: The volumetric strain-axial strain relationship curve of each scheme.

progressed, plastic failure occurred in each granule group of the coarse-grained materials; due to the different gradation composition of each scheme, Poisson's ratio of each scheme gradually differed, resulting in obvious differences in Poisson's ratio of the granule group of each scheme during failure.

4.3. Strength Analysis. Since the 20 groups of numerical specimens generated in the same scheme have different particle group distribution, under the same scheme, the calculation results of these specimens are not consistent considering coarse-grained material samples with the same



Figure 13: The relationship between $E$ and $d_{\max }$.

grading composition but different initial structure. Figure 15 shows the mean value of the peak stress of each scheme, with the increase of confining pressure, and the peak stress of coarse-grained material also increases. By analyzing the variation curves of the four peak stresses, it can be found that the mean value of the peak stresses in schemes SS1 and SS6 decreases successively when the confining pressures are the same, and with the increase of the confining pressures, the degree of the decline of the peak stresses also increases from $18.78 \%$ to $19.24 \%$. This also indicates that the scale effect of coarse-grained materials may be more obvious under highstress confining pressure, which means under high stress, the difference between the strength before and after the scaling of coarse-grained materials will be larger.

The shear strength parameters of the simulated samples were determined according to the Mohr-coulomb strength envelope, and the data of 480 triaxial simulation tests in six 




Figure 14: Tangent Poisson's ratio-axial strain relationship curve for each scheme.

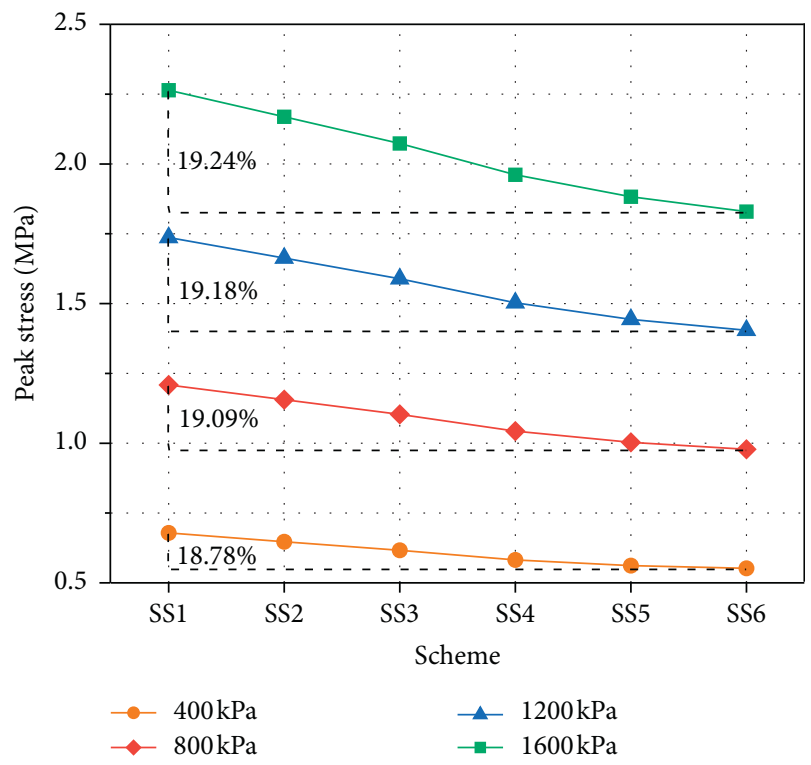

FIGURE 15: The mean value of peak stress in each scheme.

schemes were sorted out to obtain the relation between $d_{\max }$ of each scheme and the value range of its corresponding cohesive force and internal friction angle after scaling. Based on the mean value of cohesion force $\bar{c}$ and internal friction angle $\bar{\phi}$ of each scheme, the expressions of relations between $d_{\max }$ and $\bar{c}$ and between $d_{\max }$ and $\bar{\phi}$ were fitted, respectively, as shown in Figure 16.

According to Figure 16, with the increase of $d_{\max }$, the internal friction angle $\varphi$ of coarse granules increases significantly, and the mean value of internal friction angle $\bar{\phi}$ linearly grows. The value of cohesive force $c$ first decreases and then increases, and the relationship between its mean value $\bar{c}$ and $d_{\max }$ can be expressed by a unary cubic polynomial. The above relations can provide a reference for estimating the strength parameter value of the natural graded coarse-grained material. Besides, with the increase of $d_{\text {max }}$, the calculated $c$ value varies greatly, and the range of corresponding $c$ values also increases successively, which also indicates that the strength parameter $c$ of coarse-grained material is greatly affected by the scale, and the $c$ value of coarse-grained material matched with the same grade is also greatly affected by the different distribution of each granule group.

In the simulation process of the triaxial test, the influence of factors such as sample density and void ratio on the strength of coarse-grained materials in the actual sample preparation process was ignored. At the same time, the six grading curves shrunk by the parallel grading method have the same $\mathrm{Cu}, \mathrm{Cc}$ and mass fractal dimension, which minimizes the influence of other unconsidered parameters on the strength parameters of coarse-grained material. On this basis, the parameter $d_{\max }$ was used to describe 6 groups of coarse-grained materials after scaling, and the relationship between $d_{\max }$ and the shear strength parameters of coarsegrained materials is also more referential.

4.4. Sample Failure Process. As coarse-grained materials is a kind of loose geotechnical material, the sample for largescale triaxial test needs to be fixed in the rubber film before the test is carried out, as shown in Figures 4(c) and 4(d), so it is difficult to observe the damage of coarse-grained material in the process of testing. Therefore, this paper intended to use numerical simulation to show the change of the failure condition of coarse-grained material in the test process, and analyze the formation of shear failure zone.

Figure 17 shows the distribution of each particle group of the numerical sample and the calculated shear strain increment cloud diagram under a confining pressure of $1600 \mathrm{kPa}$. It can be seen that the location of shear stress concentration is mostly near the fine particle group. With the increase of the axial displacement, the shear stress further increases, the plastic zone gradually expands near the lower-strength fine particle group, and the coarse particle group appears rigid displacement.

In order to facilitate observation, the plane strain model of coarse-grained materials was established. The size of the model is $10 \mathrm{~m}$ (diameter) $\times 10 \mathrm{~m}$ (height), the confining pressure is $400 \mathrm{kPa}$, and the loading rate on the top surface of the model is $6 \times 10^{-5} \mathrm{~m} / \mathrm{s}$ in the form of axial displacement.

According to the progressive evolution of the shear failure zone in Figure 18, by neglecting particle migration and fragmentation, the formation of the shear failure zone can be divided into two stages: stress conduction stage (Figures 18(a)18(d)) and stress concentration stage (Figures 18(e)-18(h)). As the axial stress gradually increased, the stress spread from top to bottom in the specimen, and the shear stress generated inside the specimen expanded from top to bottom. This stage is the stress conduction stage. After the stress conduction reached the bottom, it began to gather at the corners of the specimen. Then, the shear stress chain at the opposite corners was connected, gathered, and continuously increased, and finally, an X-shaped 


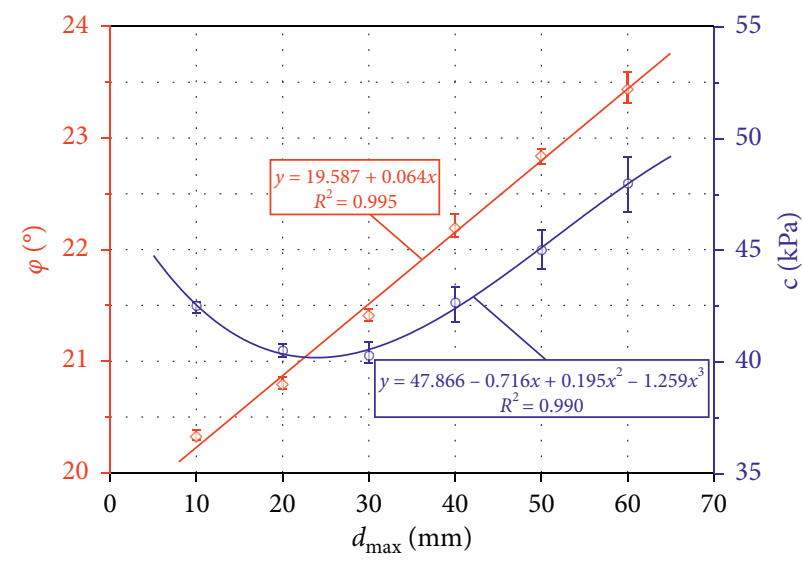

$\diamond$ Mean value of internal friction angle $\bar{\varphi}$

- Mean value of cohesion $\bar{c}$

FIGURE 16: Relationship between $d_{\max }$ and strength parameters of coarse-grained material.

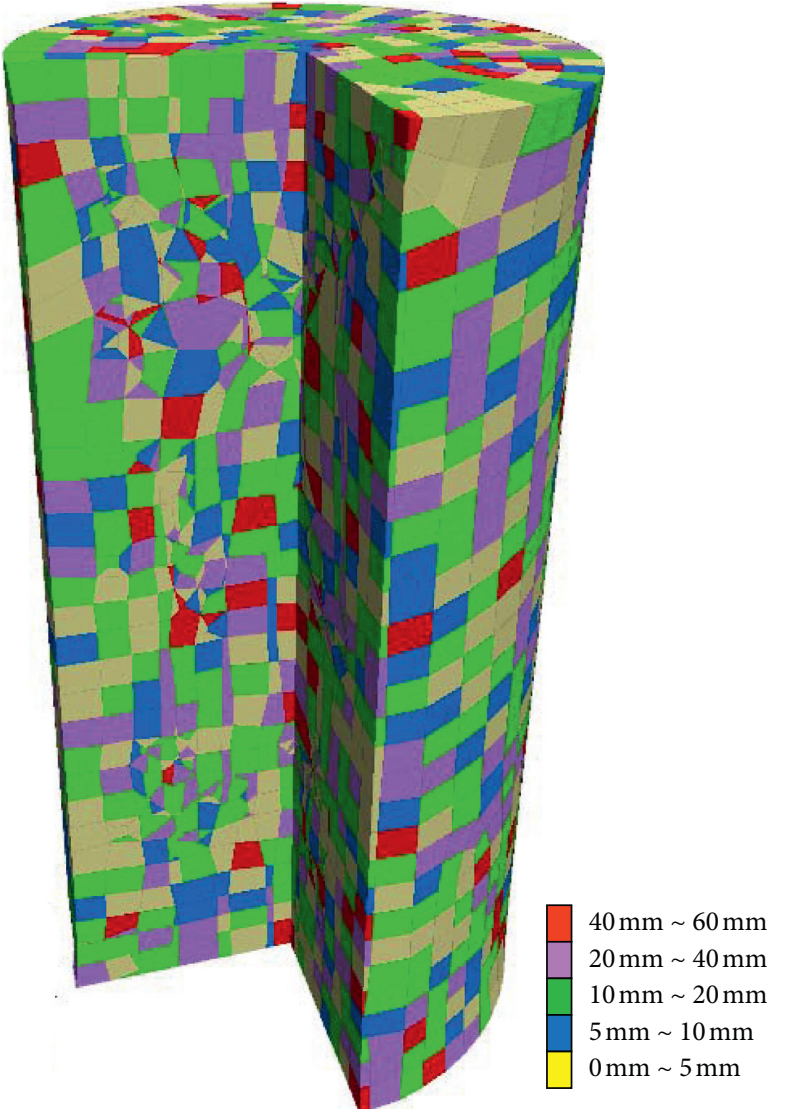

(a)

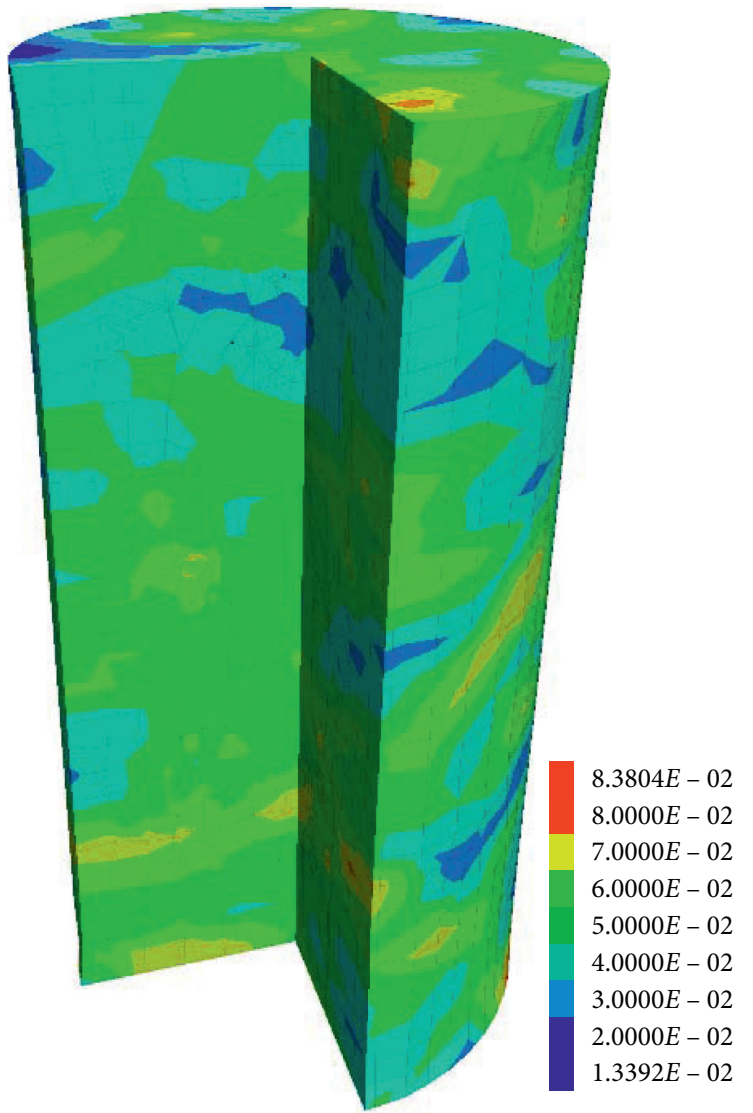

(b)

FIGURE 17: The distribution of grain groups and the cloud graph of shear strain increment: (a) the distribution of grain groups; (b) the cloud graph of shear strain increment.

shear stress concentration area, namely, the shear failure zone during the failure of the specimen, was formed. This stage is the stress concentration stage. During the stress transmission stage, the coarse-grained material mainly undergoes elastic compression, and the shear stress begins to concentrate on the surface of the weaker particles, but the sample deformation is not obvious. After entering the stress concentration stage, the shear stress concentration area is gradually obvious, until the $\mathrm{X}$-shaped shear failure zone is formed, the sample begins to undergo plastic failure, and the particles outside the shear zone begin to deform significantly along the slope. Since FLAC ${ }^{3 \mathrm{D}}$ cannot consider the breakage and migration of particles, the 




FIGURE 18: Formation of shear failure zone of numerical specimens of coarse-grained materials.

simulation results of coarse-grained materials are close to the failure of rocks. In the actual test, the failure process of the sample is basically the same as the above description. The looseness of the coarse-grained material will cause the sample first to appear many microcracks under the axial pressure, and then the microcracks gradually expand, become obvious, and finally form an obvious shear failure zone. As the coarsegrained materials are slipping along the shear failure zone, the failure deformation was extended to a certain level because it was wrapped by the rubber film and end effect. Finally, the failure shape of the specimen was formed with axial compression and bulging in the middle (as shown in Figure 4(d)).

Based on the organic combination of software SEPOHM and FLAC $^{3 \mathrm{D}}$, this paper proposes a simulation method for the triaxial test of coarse-grained materials based on laboratory tests, which can effectively characterize the anisotropy in the strength of coarse-grained materials and the randomness and nonuniformity of particle distribution in each particle group. The evolution of the shear failure zone and the scale effect of coarse-grained materials are also studied. However, it is limited by time and test conditions. On the one hand, the grid in the coarse-grained numerical specimen is used as a cell to simulate particles of different particle groups. The grid size is different from the actual granule group, and it only qualitatively describes the randomness and unevenness of the spatial distribution of each granule group based on the content. On the other hand, this paper only studies a specific coarse-grained material, but the strength or shape of the particle will affect the strength of the coarse-grained material. In the numerical simulation, the above factors need to be considered for more in-depth research. Nevertheless, this paper still has certain reference significance for the study of the numerical simulation, mechanical behavior, and scale effect of coarse-grained materials.

\section{Conclusions}

This paper simulated the discontinuity and randomness of the particle distribution of each group of coarse-grained materials through SEPOHM and realized the visualization of the distribution. After combining SEPOHM and FLAC ${ }^{3 \mathrm{D}}$, the triaxial test of coarse-grained materials was simulated by obtaining the material parameters through geotechnical tests. By comparing with the results of laboratory tests, it is found that this method can better simulate the discontinuous, nonuniform, and heterogeneous characteristics of loose geotechnical materials, to consider the anisotropy of coarse-grained materials. It provides an idea for the numerical simulation of heterogeneous geotechnical materials. Based on the above method, this paper has carried out related research studies on the scale effect of coarse-grained materials and obtained the following conclusions:

(1) Six numerical specimens with different gradations were prepared with the parallel grading method. The results showed that without considering the particle breakage and migration during the test, under the same confining pressure, the peak stress and the initial deformation modulus $E i$ of coarse-grained material continuously decrease with the decrease of $d_{\max }$. When the confining pressure increases, the strength difference before and after the scale of coarse-grained material will also increase.

(2) The volumetric strain of the numerical samples of each scheme under high-stress $(1600 \mathrm{kPa})$ conditions all appeared shear shrinkage. With the decrease of $d_{\max }$, the degree of shear shrinkage and $v$ of the sample gradually increase, and $E$ gradually decreases; $\varphi$ increases linearly with the increase of $d_{\max }$, while $c$ fluctuates with the increase of $d_{\max }$. The empirical relations between $d_{\max }$ and $\bar{c}$ and between $d_{\max }$ and $\bar{\phi}$ are presented, respectively, which can provide a reference for determining the shear strength parameters of the original-graded coarse-grained materials according to the characteristics of the grading curve.

(3) The shear stress of coarse-grained materials is mainly concentrated near the low-strength fine particle group. The formation of shear failure zone can be divided into two stages: stress conduction stage and stress concentration stage. The shear failure zone is $\mathrm{X}$-shaped when the specimen is destroyed. In the actual test, due to the end effect and the restraint of the rubber film, the specimen will be axially compressed and bulged in the middle after being damaged.

\section{Data Availability}

Research data can be seen in the relevant images and tables in the text.

\section{Conflicts of Interest}

The authors declare that they have no conflicts of interest.

\section{Acknowledgments}

The work was financially supported by the National Key Research and Development Plan (no. 2017YFC0804600); the 
Open Research Fund of State Key Laboratory of Geomechanics and Geotechnical Engineering, Institute of Rock and Soil Mechanics, Chinese Academy of Sciences (no. Z018017); and the National Natural Science Foundation of China (no. U1802243, no. 51904040, and no. 41672317).

\section{References}

[1] M. He, Z. Zhang, J. Zheng, F. Chen, and N. Li, "A new perspective on the constant $\mathrm{mi}$ of the hoek-brown failure criterion and a new model for determining the residual strength of rock," Rock Mechanics and Rock Engineering, vol. 53, no. 9, pp. 3953-3967, 2020.

[2] G. J. Wang, Y. W. Gao, and Y. J. Tang, "Research on the mechanism for chemical clogging and its effect on the stability of tailing dam," Bulgarian Chemical Communications, vol. 49, no. 1, pp. 228-233, 2017.

[3] Y. G. Zhang, J. Tang, and Z. Y. He, "A novel displacement prediction method using gated recurrent unit model with time series analysis in the Erdaohe landslide," Natural Hazards, vol. 105, pp. 783-813, 2020.

[4] L. Qiu, D. Song, Z. Li, B. Liu, and J. Liu, "Research on AE and EMR response law of the driving face passing through the fault," Safety Science, vol. 117, pp. 184-193, 2019.

[5] Y. G. Zhang and L. N. Yang, "A novel dynamic predictive method of water inrush from coal floor based on gated recurrent unit model," Natural Hazards, vol. 103, 2020.

[6] G. J. Wang, S. Tian, and B. Hu, "An experimental study on tailings deposition characteristics and variation of tailings dam saturation line," Geomechanics and Engineering, vol. 23, no. 1, pp. 85-92, 2020.

[7] Y. G. Zhang, Z. Zhang, and S. Xue, "Stability analysis of a typical landslide mass in the Three Gorges Reservoir under varying reservoir water levels," Environmental Earth Sciences, vol. 79, no. 1, 2020.

[8] G. J. Wang, J. W. Kang, and C. Du, "Study on tailings dam over-topping failure model test and break mechanism under the rainfall condition," Tehnicki Vjesnik-Technical Gazette, vol. 24, no. 6, pp. 1897-1904, 2017.

[9] G. J. Wang, S. Tian, and B. Hu, "Evolution pattern of tailings flow from dam failure and the buffering effect of debris blocking dams," Water, vol. 11, no. 11, 2019.

[10] X. T. Ai, G. J. Wang, and C. Zhang, "Research on the stability of high dump with wide graded waste rock," Rock and Soil Mechanics, vol. 41, no. 11, pp. 3777-3788, 2020.

[11] H. Z. Wei, W. J. Xu, and X. F. Xu, "Mechanical properties of strongly weathered rock-soil mixtures with different rock block contents," International Journal of Geomechanics, vol. 18, no. 5, p. 12, 2018.

[12] C. Zhang, C. Ma, Q. Chen et al., "Influence of rock percentage on strength and permeability of tailing-waste rock mixtures," Bulletin of Engineering Geology and the Environment, vol. 80, no. 1, pp. 399-411, 2021.

[13] S. L. Huang, X. L. Ding, and Y. T. Zhang, "Triaxial test and mechanical analysis of rock-soil aggregate sampled from natural sliding mass," Advances in Materials Science and Engineering, vol. 2015, p. 14, 2015.

[14] Y. Xiao, H. L. Liu, and B. W. Nan, "Gradation-dependent thermal conductivity of sands," Journal of Geotechnical and Geoenvironmental Engineering, vol. 144, no. 9, p. 5, 2018.

[15] G. Yang, Y. Jiang, and S. Nimbalkar, "Influence of particle size distribution on the critical state of rockfill," Advances in Civil Engineering, vol. 2019, p. 7, 2019.
[16] K. M. Wei, S. Zhu, and X. H. Yu, "Influence of the scale effect on the mechanical parameters of coarse-grained soils," Iranian Journal of Science and Technology-Transactions of Civil Engineering, vol. 38, no. C1, pp. 75-84, 2014.

[17] A. Hamidi, E. Azini, and B. Masoudi, "Impact of gradation on the shear strength-dilation behavior of well graded sandgravel mixtures," Scientia Iranica, vol. 19, no. 3, pp. 393-402, 2012.

[18] W. L. Wu, Z. X. Tu, and Z. H. Zhu, "Effect of gradation segregation on mechanical properties of an asphalt mixture," Applied Sciences-Basel, vol. 9, no. 2, p. 15, 2019.

[19] Y. Huang, J. Li, Y. Teng et al., "Numerical simulation study on macroscopic mechanical behaviors and micro-motion characteristics of gangues under triaxial compression," Powder Technology, vol. 320, pp. 668-684, 2017.

[20] F. Yan, X. Feng, P. Pan, and S. Li, "Rock initiation and propagation simulation under compression-shear loading using continuous-discontinuous cellular automaton method," Acta Mechanica Solida Sinica, vol. 28, no. 4, pp. 384-399, 2015.

[21] N. Prosperini and D. Perugini, "Application of a cellular automata model to the study of soil particle size distributions," Physica A: Statistical Mechanics and Its Applications, vol. 383, no. 2, pp. 595-602, 2007.

[22] F. Barrio-Parra, M. Izquierdo-Diaz, and L. J. FernandezGutierrez del Alamo, "Modelling the transference of trace elements between environmental compartments in abandoned mining areas," International Journal of Environmental Research and Public Health, vol. 17, no. 14, 2020.

[23] G. J. Wang, X. Y. Kong, and C. H. Yang, "Research on the representation of granular vertical size grading and numerical simulation method with the typical waste rock site," Tehnicki Vjesnik-Technical Gazette, vol. 22, no. 3, pp. 677-683, 2015.

[24] D. P. Xu, B. Wang, and L. J. Jiang, "Study of methods of triaxial numerical simulation test of glacial till," Rock and Soil Mechanics, vol. 29, no. 12, pp. 3466-3470, 2008.

[25] G. J. Wang, X. Y. Kong, and C. H. Yang, "Research on the slope stability considering random distribution and size grading of granular in the dumping site," Disaster Advances, vol. 6, pp. 102-107, 2013.

[26] Q. G. Guo, Engineering Characteristics and Application of Coarse-Grained Soil, The Yellow River Water Conservancy Press, Jinan, China, 1998. 